\title{
The prognostic effect of brain natriuretic peptide levels on outcomes of hospitalized patients with COVID-19
}

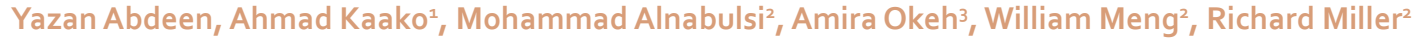 \\ Pulmonary and Sleep Physicians of Houston, PA, ${ }^{1}$ Mercy Hospital Fort Smith, Fort Smith, AR, ${ }^{2}$ Saint Michael's Medical Center, Newark, NJ, \\ ${ }^{3}$ University of Illinois at Chicago, Chicago, IL, USA
}

\begin{tabular}{|c|}
\hline Access this article online \\
\hline Website: www.avicennajmed.com \\
\hline DOI: 10.4103/ajm.ajm_169_20 \\
\hline Quick Response Code: \\
\hline
\end{tabular}

\begin{abstract}
Natriuretic peptides are biomarkers of myocardial stress and are frequently elevated among patients with severe respiratory illnesses, typically in the absence of elevated cardiac-filling pressures or clinical heart failure. Elevation of brain natriuretic peptide (BNP) or NT-proBNP is associated with worse outcomes among patients with Acute Respiratory Distress Syndrome (ARDS). We conducted a retrospective cohort study based on a comprehensive review of Electronic Medical Records (EMRs) of patients with Coronavirus Disease 2019 (COVID-19) to evaluate whether BNP on admission has prognostic value on mortality and hospital length of stay (LOS) among patients admitted with confirmed COVID-19 along with the inclusion of additional prognostic variables. Overall, 146 patients were included after analyzing 230 patients' EMR and excluding potential confounding factors for abnormal BNP. Our statistical analysis did not show a statistically significant association between BNP level and mortality rate $(P=0.722)$ or ICU LOS $(P=0.741)$. A remarkable secondary outcome to our study was that impaired renal function (GFR $<60$ ) on admission was significantly associated with an increased mortality rate $(P=0.026)$ and an increased ICU LOS $(P=0.022)$. Although various studies have presented the predictive role of pro-BNP among patients with respiratory distress in the past years, our study did not find BNP to be an accurate predictive and prognostic factor among patients with COVID-19 in our study population. Renal impairment and high Acute Physiology and Chronic Health Evaluation (APACHE) II scores on admission, on the other hand, have demonstrated to be strong predictors for COVID-19 morbidity and mortality. This study could represent an introduction to more prominent multicenter studies to evaluate additional prognostic factors and minimize the ordering of nonspecific testing.
\end{abstract}

Key words: Brain natriuretic peptide, COVID-19, SARS-CoV-2

\section{INTRODUCTION}

COVID-19, a disease caused by the severe acute respiratory syndrome coronavirus 2 (SARS-CoV-2) that belongs to the Coronaviruses family, has been one of the most devastating outbreaks that led to the first Coronavirus pandemic in the 21 st century. ${ }^{[1]}$ SARS-CoV-2 is a highly infectious virus that could result in significant morbidity and mortality, as reported by the Centers for Disease Control and Prevention

Address for correspondence: Dr. Ahmad Kaako,

Hospitalist Physician, Mercy Hospital, Physicians Building,

7300 Rogers Ave, Fort Smith, AR.

E-mail: ahmadkaako@hotmail.com
(CDC). ${ }^{[2]}$ SARS-CoV-2 mainly affects the respiratory system, resulting in a spectrum of illnesses ranging from Upper Respiratory Tract Infections to Severe Acute Respiratory Syndrome (SARS).$^{[1,3]}$ Due to the devastating effects of

This is an open access journal, and articles are distributed under the terms of the Creative Commons Attribution-NonCommercial-ShareAlike 4.0 License, which allows others to remix, tweak, and build upon the work non-commercially, as long as appropriate credit is given and the new creations are licensed under the identical terms.

For reprints contact: reprints@medknow.com

Cite this article as: Abdeen Y, Kaako A, Alnabulsi M, Okeh A, Meng W, Miller $R$. The prognostic effect of brain natriuretic peptide levels on outcomes of hospitalized patients with COVID-19. Avicenna J Med 2021;11:20-6. 
COVID-19 on the health-care system, prognostication has become crucial for proper resource allocation and triaging patients with COVID-19 according to their risks. Therefore, more focus has been directed toward identifying COVID-19 infection, including patient factors and diagnostic tests, to identify patients with higher risk. ${ }^{[4,5]}$ Natriuretic peptides are biomarkers of myocardial stress and are frequently elevated among patients with severe respiratory illnesses, typically in the absence of elevated cardiac-filling pressures or clinical heart failure. ${ }^{[6]}$ Elevation of BNP or NT-proBNP is associated with worse outcomes among patients with ARDS. ${ }^{[7]}$ Therefore, this study was conducted to identify the prognostic value of BNP among hospitalized patients with SARS-CoV-2 infection.

\section{METHODS}

\section{The study design and oversight}

This study is a retrospective cohort study based on a comprehensive review of electronic medical records (EMRs). It was designed by the principal investigator. All of the authors reviewed the manuscript before submission for publication. The approval of the Institutional Review Board (IRB) (\#16/20) was issued before data collection. The study variables were extracted from the EMR system (EPIC at Saint Michael's Medical Center) and were inserted directly into and stored in a password-protected EXCEL worksheet available only to the study investigators. No paper files were printed or stored. All the data were kept private and confidential per IRB and HIPAA policies. Based on the study's retrospective nature, the patients' informed consent requirement was waived by the IRB.

\section{The study population}

Saint Michael's Medical Center (SMMC) is an inner-city community hospital located in Newark, New Jersey, and affiliated with New York Medical College. It serves an area with a Latino and African American majority. The SMMC hosted a significantly number of patients with COVID-19 during the pandemic. The EMRs were screened for all the adult patients (age $\geq 18$ years) and admitted to SMMC with respiratory distress and confirmed COVID-19 infection between 1 February 2020 and 30 April 2020.

\section{Study outcomes}

The primary outcomes were in-hospital mortality and admission to the intensive care unit (ICU) with mechanical ventilation and the hospital LOS. Our primary goal was to evaluate whether BNP on admission has prognostic value for the earlier-mentioned outcomes among patients admitted with confirmed COVID-19. Our secondary goal was to evaluate any other potential prognostic variables that may predict outcomes associated with COVID-19 infection.

\section{Definitions}

A fatal case of COVID-19 was defined as any death that occurred during hospitalization with a confirmed diagnosis of COVID-19 made by PCR testing of nasopharyngeal swab samples. A non-fatal case was defined as a confirmed case of COVID-19 by PCR in a hospitalized patient who had not died (whether discharged or still hospitalized) as of 30 April 2020. Due to the limited intensive care unit (ICU) bed availability during the pandemic and the state of emergency declaration, almost all the ICU patients were on mechanical ventilation. Almost all the extubated patients were transferred out of the ICU on the same day, a few hours after extubation. Hence, the time of mechanical ventilation was used as a proxy estimate for the ICU length of stay (LOS), which was not measured directly in our study. The hospital LOS was calculated (in days) by subtracting the date of admission from the day of discharge.

\section{Inclusion criteria}

The main inclusion criteria were as follows:

1. Adult patients (age $\geq 18$ years) presenting with active respiratory symptoms related to SARS-CoV-2 infection, including shortness of breath, cough along with oxygen saturation less than $92 \%$ on room air.

2. Confirmed case of COVID-19 by PCR.

3. Patients had blood BNP levels drawn and reported on the day of admission.

Clinically, some medical conditions may independently alter BNP levels in patients with normal heart function and no respiratory distress ${ }^{[6]}$ To minimize confounding factors that may affect our evaluation of the dependent variable (BNP) effect on the primary outcomes, we excluded patients with a known history of End-Stage Renal Disease (ESRD), GFR $\leq 15 \mathrm{~mL} / \mathrm{min}$, or patients with morbid obesity (BMI $\geq 40 \mathrm{~kg} /$ $\mathrm{m}^{2}$ ) on presentation since these medical conditions may independently alter the BNP levels. ${ }^{[6]}$

\section{Methods}

EMRs were reviewed to extract the study variables, which included demographics (age, sex, and race), BMI, Ejection Fraction, GFR on admission, and comorbidities, including Diabetes Mellitus (DM), Chronic Obstructive Pulmonary Disease (COPD), and ESRD. A total of 230 patients were identified to be admitted with respiratory distress and positive COVID-19 in the defined period. One hundred forty-six patients were included in the final analysis. 
Cases excluded were as follows: 22 patients had no BNP drawn on admission, 13 patients had a history of ESRD, 10 patients presented with GFR $\leq 15 \mathrm{~mL} / \mathrm{min}$ on admission, 21 patients had $\mathrm{BMI} \geq 40 \mathrm{~kg} / \mathrm{m}^{2}$ on admission, and eight patients had no EF available. APACHE II score; a known predictor of mortality, ${ }^{[8]}$ was calculated for all the included subjects by using the SFAR scoring website ${ }^{[9]}$ and number recorded on our datasheet.

The primary endpoints or outcomes were to determine the in-hospital mortality outcome as well as the duration of mechanical ventilation for patients admitted to the ICU (in days) and the hospital LOS (in days) for all subjects. To evaluate the effect of some continuous variables on the outcomes of interest, we classified the subjects into subgroups. We classified the patients based on the BNP value into two groups: one with normal BNP (defined as BNP $\leq$ $100 \mathrm{pg} / \mathrm{mL}$ ) and the other with high BNP (defined as BNP $>100 \mathrm{pg} / \mathrm{mL}$ ). All the patients with available prior or current echocardiogram reports were classified into two subgroups based on the ejection fraction ( $\mathrm{EF} \geq 50 \%$ vs. $<50 \%$ ). Further, all the patients were classified into two subgroups based on the GFR (GFR $\geq 60 \mathrm{~mL} / \mathrm{min}$ vs. GFR $<60 \mathrm{~mL} / \mathrm{min}$ ).

\section{Statistical analysis}

We described the cohort characteristics by using frequency tables with proportions for binary and categorical variables and mean (with standard deviation) or median (with interquartile ranges) for the continuous variables based on the distribution. We reported the descriptive statistics for all the patients and then categorized them into two subgroups (patients with fatal cases and patients with nonfatal cases) based on the death outcome. We analyzed the binary, categorical, and continuous variables between the two subgroups and measured the association between the two, by using Pearson $\chi^{2}$-test for binary and categorical variables and $t$-test or nonparametric tests for continuous variables, as appropriate. We examined the association between the multiple variables and both hospital and ICU LOS by conducting univariant (unadjusted) and multivariant (adjusted) regression analyses. The highly correlated variables were removed from the regression analyses models to avoid collinearity. A $P$-value of $<0.05$ is considered statistically significant. All calculations were made by using STATA version 14.2 (StataCorp, College Station, TX, USA).

\section{RESULTS}

A total of 230 patients were identified to be admitted with respiratory symptoms, including dyspnea, cough, and hypoxia related to a confirmed diagnosis of COVID-19.
One hundred forty-six patients were included in the final analysis.

Table 1 shows the demographic and clinical characteristics of the patients. Overall, $61.6 \%$ of patients were men, and the mean $( \pm \mathrm{SD})$ age was $60.8 \pm 15.1$ years. More than half (56.2\%) of the patients were Hispanic, and the remainder as outlined in Table 1 . The mean $( \pm$ SD) BMI was $28.7 \pm 5.24$, and $44.5 \%$ of the patients had obesity. Almost one-quarter of the patients (24\%) had GFR $<60$ (keeping in mind that patients with ESRD and GFR $\leq 15$ were excluded). Almost one-third of the patients (30.1\%) had a history of DM, and only a small fraction (6.2\%) had a history of COPD. Most patients (93.1\%) had no history of cardiomyopathy with documented $\mathrm{EF} \geq 50 \%$, and only $6.9 \%$ of the patients had a documented $\mathrm{EF}<50 \%$. The average $( \pm \mathrm{SD})$ hospital LOS for all the patients was $8.38 \pm 6.33$ days. The average ( \pm SD) ICU LOS was $9.94 \pm 6.7$ days for the critical patients who required ventilation and ICU admission. The mean APACHE II score $( \pm$ SD) on admission was $11.5 \pm 5.6$. The differences between the two subgroups based on the mortality outcome are listed in Table 1.

Based on the lack of normal distribution for the BNP values, the median and interquartile range were reported, and the Wilcoxon rank-sum (Mann-Whitney) test was used to compare the two subgroups. Although the P-value was consistent with a statistically significant difference between the two subgroups, it was not clinically relevant as the statistical difference was accounted for by the significant variation in the normal values $(B N P<100)$ in the two subgroups. When the comparison was performed, using a value of BNP > 100, there was no statistically significant difference between the two subgroups. Patients in the group of GFR $<60$ had significantly higher rates of death compared with the group of GFR $60(P=0.026)$. APACHE II score on admission was a reliable prognostic indicator for the outcome of death, with a remarkably higher APACHE II score on admission in patients with fatal cases vs patients with nonfatal cases $[15.5 \pm 6.4$ vs. $10.7 \pm$ 5.1 respectively, with $P=0.001$ ]. There was no statistically significant difference between the two subgroups (patients with and without in-hospital mortality) regarding age, gender, race, obesity, DM, COPD, EF subgroups, BMI, and hospital LOS.

We examined the association between hospital LOS as an outcome with each of the appropriate variables, as shown in Table 2. Age, BMI, and APACHE II score were added as continuous variables. Sex, race, GFR, COPD, DM, and EF subgroups were all added as binary variables with male, Hispanic race, normal GFR, no COPD, no DM, and normalEF 


\begin{tabular}{|c|c|c|c|c|}
\hline Variable & All patients & $\begin{array}{l}\text { Patient with in-hospital } \\
\text { mortality }\end{array}$ & $\begin{array}{l}\text { Patient without in-hospital } \\
\text { mortality }\end{array}$ & $P$-value \\
\hline Mean age (SD)-year & $60.79(15.11)$ & $64.08(16.11)$ & $60.147(14.90)$ & 0.27 \\
\hline Male & $59.9(14.68)$ & $61.526(15.689)$ & $59.52(14.48)$ & \\
\hline Female & $62.16(15.824)$ & $73.8(15.32)$ & 61.02 (15.55) & \\
\hline Sex: no./total no. (\%) & & & & 0.053 \\
\hline Male & $90 / 146(61.6 \%)$ & 19/24 (79\%) & $71 / 122$ (58\%) & \\
\hline Female & $56 / 146(38.36 \%)$ & $5 / 24(21 \%)$ & $5 \mathrm{I} / \mathrm{I} 22(42 \%)$ & \\
\hline \multicolumn{5}{|l|}{ Race: no./total no. (\%) } \\
\hline Hispanic & $82 / 146(56.16 \%)$ & $13 / 24(54 \%)$ & $69 / 122(57 \%)$ & \\
\hline African American & $20 / 146(13.7 \%)$ & $4 / 24(17 \%)$ & $16 / 122(13 \%)$ & 0.896 \\
\hline Other & $2 / 146$ (1.37\%) & 0 & $2 / 122(2 \%)$ & \\
\hline Missing data & $42 / 146$ (28.77 \%) & $7 / 24(29 \%)$ & $35 / / 22(29 \%)$ & \\
\hline BMI—mean (SD) & $28.7(5.24)$ & $29.6(5.1)$ & $28.5(5.27)$ & 0.35 \\
\hline Obesity BMI [30-40] & $65 / 146$ (44.5\%) & $10 / 24(42 \%)$ & $55 / 122(45 \%)$ & 0.758 \\
\hline Median BNP (IQ range) & $22.5(8-60)$ & $50.5(14.5-175.5)$ & $21(6-48)$ & 0.011 \\
\hline BNP $>100$ & $256(169.5-808)$ & $374(175.5-876)$ & $24 I(\mid 59.5-808)$ & 0.722 \\
\hline GFR group & & & & 0.026 \\
\hline$\geq 60$ & $111 / 146(76 \%)$ & | $4 / 24$ (58\%) & $97 / 122(80 \%)$ & \\
\hline$<60$ & $35 / 146(24 \%)$ & $10 / 24(42 \%)$ & $25 / 122(20 \%)$ & \\
\hline DM & $44 / 146$ (30.14\%) & $6 / 24(25 \%)$ & $38 / 122(31 \%)$ & 0.549 \\
\hline COPD & $9 / 146(6.2 \%)$ & $2 / 24(8 \%)$ & $7 / 122(6 \%)$ & 0.629 \\
\hline EF group & & & & 0.569 \\
\hline$\geq 50$ & $136 / 146(93.15 \%)$ & $23 / 24(96 \%)$ & I I $3 / 122$ (92\%) & \\
\hline$<50$ & $10 / 146(6.85 \%)$ & $1 / 24(4 \%)$ & $9 / 122(7 \%)$ & \\
\hline ICU LOS (if applicable) & $9.94(6.7)$ & $7.54(4.88)$ & $12.45(6.48)$ & 0.01 \\
\hline Average hospital LOS (SD): day & $8.38(6.33)$ & $9.4(5.3)$ & $8.2(6.5)$ & 0.32 \\
\hline Mean APACHE II score (SD) & $\mathrm{II} .5(5.6)$ & $15.5(6.4)$ & $10.7(5.1)$ & 0.0014 \\
\hline
\end{tabular}

\begin{tabular}{|c|c|c|c|c|}
\hline \multirow[t]{2}{*}{ Variable } & \multicolumn{2}{|c|}{ Univariant unadjusted model } & \multicolumn{2}{|c|}{ Multivariant adjusted model* } \\
\hline & Coef. $(95 \% \mathrm{Cl})$ & P-value & Coef. $(95 \% \mathrm{Cl})$ & P-value \\
\hline Age (year) & $-0.006(-0.075-0.06)$ & 0.861 & $-0.58(-0.14-0.028)$ & 0.184 \\
\hline Sex & $-0.466(-2.6-1.67)$ & 0.667 & $-0.33(-2.48-1.8)$ & 0.758 \\
\hline Race & $0.08(-1.054-1.21)$ & 0.892 & $.198(-.98-1.37)$ & 0.739 \\
\hline BMI & $0.04(-0.16-0.24)$ & 0.714 & $.0185523(-0.19-0.23)$ & 0.860 \\
\hline BNP & $0.0008(-0.001-0.003)$ & 0.422 & $.0003(-.002-0.002)$ & 0.741 \\
\hline GFR group & $-0.65(-3.08-1.79)$ & 0.600 & $-3.57(-6.62-0.52)$ & 0.022 \\
\hline DM & $-0.21(-2.48-2.05)$ & 0.852 & $-0.67(-3.05-1.7 I)$ & 0.579 \\
\hline COPD & $-0.17(-4.49-4.16)$ & 0.940 & $-0.34(-4.75-4.06)$ & 0.878 \\
\hline EF group & $0.5617647(-3.56-4.68)$ & 0.788 & $1.07(-03.29-5.42)$ & 0.629 \\
\hline APACHE II score & $0.2067085(.025-0.39)$ & 0.026 & $0.46(0.2-0.7 I)$ & 0.001 \\
\hline
\end{tabular}

*The model includes age, sex, race, BMI, BNP, GFR group, EF group, DM, COPD, and APACHE II score.

used as reference groups, respectively. The highly correlated variables were removed from the regression analysis models to avoid collinearity. The variable obesity was excluded, as BMI (as a continuous variable) was included as an independent variable. Adding obesity as a binary variable $(\mathrm{BMI}>30)$ after excluding the continuous BMI variable did not yield any statistically significant difference. The ICU LOS was removed from the model that predicts the hospital LOS (and vice versa) due to their potential collinearity.

In the univariant regression analyses, only the APACHE II score showed a strong association with the hospital LOS $(P=0.026)$. On adjustment with multivariant regression analysis to predict hospital LOS, APACHE II remained a strong predictor of the hospital LOS after adjustment $(P=0.001)$. Further, the GFR group was also strongly associated with hospital LOS $(P=0.22)$. None of the other variables had any predictive value in estimating the hospital LOS based on the univariant and multivariant analyses.

Of the total of 146 patients included in our final cohort, 46 patients were admitted to the ICU. We performed both univariant and multivariant regression analyses to evaluate the association between the ICU LOS as an outcome with each of the appropriate variables in a similar way to the hospital LOS unadjusted and adjusted models. None of the variables was a reliable predictor of the ICU LOS with the small sample size of ICU patients. 


\section{DISCUSSION}

Our study aimed at finding prognostic factors associated with more severe outcomes in patients who acquired COVID-19 infection to help providers determine patients at high risk. Identifying prognostic and predictive factors is crucial to assess various medical conditions and to help physicians anticipate clinical outcomes, especially for patients with critical conditions. ${ }^{[4,10,11]}$ Although the APACHE II scoring system is utilized mainly in critical care settings, the variety of physiologic and laboratory parameters needed for calculation may not be readily available. ${ }^{[12,13]}$ Hence, the search for other predictive and prognostic factors is rising, especially in the light of the SARS-COV-2 pandemic, which has generated vast numbers of critically ill patients in a short time..$^{[4,10,11]}$ We applied the APACHE II among our subjects, and as expected, it was shown to be a valuable tool to predict mortality and LOS among patients with COVID-19 in our study. Two recent studies have illustrated a correlation between obesity, male sex, increasing age, and DM, with worse outcomes among hospitalized patients with COVID-19 infection. ${ }^{[14,15]}$

Lang Wang et al. found that a high percentage of severe to critical cases and high mortality rates were observed in elderly patients with COVID-19. ${ }^{[16]}$ Patients' conditions on admission, such as dyspnea, lymphocytopenia, cardiovascular disease, COPD, and ARDS occurrence during hospitalization, predicted fatal outcomes. ${ }^{[16]}$ Another study by Tao Guo suggested that myocardial injury due to potential inflammation has a significant association with fatal outcomes of patients with COVID-19 due to impairment of cardiac function and ventricular tachyarrhythmias. ${ }^{[17]}$

BNP level offers a promising value for patients presenting with acute dyspnea among patients with heart failure. ${ }^{[18]}$ Studies have demonstrated that plasma BNP level on admission helps differentiate between pulmonary and cardiogenic causes of dyspnea with high specificity and negative predictive value. ${ }^{[18,19]}$ NT-proBNP is usually released from the myocardial cell wall secondary to stress, in addition to the inflammatory molecules such as lipopolysaccharide, interleukin 1, C-reactive protein, and cardiotrophin, which are independent of ventricular function. ${ }^{[10,20]}$ Moreover, Nagaya et al. have suggested that there is likely a strong correlation between BNP levels and the mean pulmonary arterial pressure, as pressure load or right ventricular strain may be the underlying pathophysiologic mechanism. ${ }^{[21,22]}$

Acknowledgment of the cardiac and noncardiac factors that affect the BNP plasma level is vital in identifying underlying pathologies and excluding confounding factors on clinical encounters. BNP has a principal and robust effect on the kidneys by promoting tubular natriuresis and diuresis. Under normal physiologic circumstances, falls in cardiac output, adequate blood volume, and renal blood flow are accompanied by activation of the renin-angiotensinaldosterone system to preserve the blood pressure and intravascular volume. The BNP effect is to oppose this effect by promoting natriuresis and increasing GFR. ${ }^{[23]}$ Renal dysfunction decreases this effect, as the kidney becomes less responsive to BNP, denoting BNP's oversecretion to compensate for the relative hypervolemia and cardiac wall stress from the overload At the same time, heart failure reduces renal function effectiveness by reducing cardiac output and potentiating adverse effects. Thus, heart failure and renal dysfunction act synergistically in their ability to increase the secretion of BNP. This pathophysiological mechanism explains the elevation of BNP concentrations seen in renal failure patients ${ }^{[24,25]}$ and resonates with what was found in previous studies that BNP concentrations were progressively higher in patients with progressively advanced CKD and ESRD. ${ }^{[26]}$ Omitting confounding factors is likewise essential to yield vigorous results and increase the significance of a study.

Some reports from cardiologists in Chongqing, China, have suggested a correlation between NT- proBNP level and an increased risk of mortality in hospitalized patients with COVID-19. ${ }^{[1]]}$

One of our study's goals was to find whether there was a correlation between elevated BNP levels and a higher risk of death after SARS-CoV-2 infection. Our statistical analysis did not find a statistically significant association between BNP level and mortality rate $(P=0.722)$ or ICU LOS $(P=0.741)$. Our study findings were similar to the previous study, which showed no correlation between BNP levels and ICU LOS in patients with dyspnea. ${ }^{[13]}$

Interestingly, a remarkable secondary outcome to our study was that impaired renal function $(G F R<60)$ on admission was significantly associated with an increased mortality rate $(P=0.026)$ and an increased ICU LOS $(P=0.022)$. In addition, patients with CKD were more likely to be admitted to the ICU and undergo mechanical ventilation. This observation suggests that kidney disease, whether acute or chronic, may represent a higher risk to faster deterioration and more significant adverse clinical outcomes. The putative receptor for SARS-CoV-2, the angiotensin-converting enzyme 2 (ACE2), is expressed in the human's kidneys, which may explain why the kidneys may be a direct target of the virus. ${ }^{[27]}$ Another plausible 
mechanism is the superimposed pulmonary edema due to impaired fluid excretion. Another possibility is the limited COVID-19 pharmacological treatment options in patients with renal impairment due to the lack of safety data among these patient groups. These findings have corresponded to some of the previous studies that kidney injury was associated with an increased risk of death in patients with influenza A virus subtype H1N1 and SARS ${ }^{[28,29]}$ as well as recent studies in patients with COVID-19 infection. ${ }^{[30]}$ Further, recent studies showed that COVID-19 virus directly infects the human kidney tubules and induces acute tubular toxicity through different direct and indirect paths that result in tubular pathophysiology. ${ }^{[1,32]}$

Our study population consisted of a high percentage of the Hispanic race, which is expected based on the Latino majority in the hospital area. There is only a small fraction of the Caucasian race in the surrounding area, which explains why it is included under "other" in Table 1.

\section{Limitations}

Our study included subjects from a single inner-city hospital. Our study population consists of a high percentage of Hispanic and African American races, which precludes generalization. Due to the strict inclusion criteria to minimize confounding factors, many subjects have been excluded from our analysis, which limited the number of our inclusion subjects. Further, all the patients who were screened and met the criteria were included in the study without a random selection process implemented, which may generate a selection bias. As echocardiograms (and other tests) were minimized during the pandemic to reduce unnecessary contact with patients with COVID-19, we used the patients' previous echocardiogram reports as a proxy to determine their ejection fraction and congestive heart failure (CHF) status on admission. Previous echocardiograms may not reflect the current CHF and ejection fraction status for patients admitted with COVID-19, which could be considered another confounder. Finally, the retrospective design of the study has its own inherited limitations.

\section{CONCLUSION}

Ongoing research is being conducted to identify prognostic factors among patients with COVID-19. Although various studies have presented the predictive role of pro-BNP among patients with respiratory distress in the past years, our study did not find BNP an accurate predictive and prognostic factor among patients with COVID-19 in our study population. Renal impairment and high APACHE II scores on admission, on the other hand, have demonstrated to be strong predictors for COVID-19 morbidity and mortality. This study could represent an introduction to more prominent multicenter studies to evaluate additional prognostic factors and minimize the ordering of nonspecific testing.

\section{Financial support and sponsorship}

Nil.

\section{Conflicts of interest}

There are no conflicts of interest.

\section{REFERENCES}

1. Mehra MR, Desai SS, Kuy S, Henry TD, Patel AN. Cardiovascular disease, drug therapy, and mortality in Covid-19. N Engl J Med 2020;382:e102.

2. Schuchat A. CDC Covid-19 Response Team. Public health response to the initiation and spread of pandemic COVID-19 in the United States, February 24-April 21, 2020. MMWR Morb Mortal Wkly Rep 2020;69:551-6.

3. Gandhi RT, Lynch JB, Del Rio C. Mild or moderate Covid-19. N Engl J Med 2020;383:1757-66.

4. Henry B, de Oliveira M, Benoit S, Plebani M, Lippi G. Hematologic, biochemical and immune biomarker abnormalities associated with severe illness and mortality in coronavirus disease 2019 (Covid-19): A meta-analysis. Clin Chem Lab Med 2020;58:1021-8.

5. Wendel Garcia PD, Fumeaux T, Guerci P, Heuberger DM, Montomoli J, Roche-Campo F, et al. Prognostic factors associated with mortality risk and disease progression in 639 critically ill patients with COVID-19 in Europe: Initial report of the international RISC-19-ICU prospective observational cohort. EClinicalMedicine 2020;25:100449.

6. Ibrahim NE, Januzzi JLJr. Established and emerging roles of biomarkers in heart failure. Circ Res 2018;123:614-29.

7. Bajwa EK, Januzzi JL, Gong MN, Thompson BT, Christiani DC. Prognostic value of plasma $\mathrm{N}$-terminal probrain natriuretic peptide levels in the acute respiratory distress syndrome. Crit Care Med 2008;36:2322-7.

8. Sekulic A, Trpkovic S, Pavlovic A, Marinkovic O, Ilic A. Scoring systems in assessing survival of critically ill ICU patients. Med Sci Monit: Int Med J Exp Clin Res 2015;21:2621-9.

9. SFAR. Scoring systems for ICU and surgical patients. Available from: https:// sfar.org/scores2/apache22.php\# haut [Last accessed on 2020 Dec 3].

10. Vallabhajosyula S, Wang Z, Murad M, Vallabhajosyula S, Sundaragiri PR, Kashani $\mathrm{K}$, et al. Natriuretic peptides to predict short-term mortality in patients with sepsis: A systematic review and meta-analysis. Mayo Clin Proc Innov Qual Outcomes 2020;4:50-64.

11. Gao L, Jiang D, Wen X, Cheng X, Sun M, He B, et al. Prognostic value of NT-proBNP in patients with severe COVID-19. Respir Res 2020;21:83.

12. Ferreira F, Bota D, Bross A, Mélot C, Vincent J. Serial evaluation of the SOFA score to predict outcome in critically ill patients. JAMA 2001;286:1754-8.

13. Abdeen Y, Sen P, Safdar S, Katapally R, Arqoob AA, Macbruce D, et al. The usefulness of brain natriuretic peptide level in diagnosis and prognosis of patients admitted to critical care unit with shortness of breath. J Emerg Trauma Shock 2015;8:205-9.

14. Palaiodimos L, Chamorro-Pareja N, Karamanis D, Li W, Zavras PD, Mathias $\mathrm{P}$, et al. Diabetes is associated with increased risk for in-hospital mortality in patients with COVID-19: A systematic review and metaanalysis comprising 18,506 patients. Hormones 2020:1-10.

15. Palaiodimos L, Kokkinidis DG, Li W, Karamanis D, Ognibene J, Arora S, et al. Severe obesity is associated with higher in-hospital mortality in a cohort of patients with COVID-19 in the Bronx, New York. Metabolism 2020;108:154262. 
16. Wang L, He W, Yu X, Hu D, Bao M, Liu H, et al. Coronavirus disease 2019 in elderly patients: Characteristics and prognostic factors based on 4-week follow-up. J Infect 2020;80:639-45.

17. Guo T, Fan Y, Chen M, Wu X, Zhang L, He T, et al. Cardiovascular implications of fatal outcomes of patients with coronavirus disease 2019 (COVID-19). JAMA Cardiol 2020;5:811-8.

18. Morrison L, Harrison A, Krishnaswamy P, Kazanegra R, Clopton P, Maisel A. Utility of a rapid B-natriuretic peptide assay in differentiating congestive heart failure from lung disease in patients presenting with dyspnea. J Am Coll Cardiol 2002;39:202-9.

19. Davis M, Espiner E, Richards G, Billings J, Town I, Neill A, et al. Plasma brain natriuretic peptide in assessment of acute dyspnoea. Lancet 1994;343:440-4.

20. Shor R, Rozenman Y, Bolshinsky A, Harpaz D, Tilis Y, Matas Z, et al. BNP in septic patients without systolic myocardial dysfunction. Eur J Intern Med 2006;17:536-40.

21. Noritoshi N, Toshio N, Masaaki U, Toru S, Shingo K, Fumio S, et al. Plasma brain natriuretic peptide as a prognostic indicator in patients with primary pulmonary hypertension. Circulation 2000;102:865-70.

22. Casserly B, Klinger J. Brain natriuretic peptide in pulmonary arterial hypertension: Biomarker and potential therapeutic agent. Drug Des, Devel Ther 2009;3:269-87.

23. Holmes S, Espiner E, Richards A, Yandle T, Frampton C. Renal, endocrine, and hemodynamic effects of human brain natriuretic peptide in normal man. J Clin Endocrinol Metab 1993;76:91-6.

24 McCullough P, Duc P, Omland T, McCord J, Nowak R, Hollander J, et al. B-type natriuretic peptide and renal function in the diagnosis of heart failure: An analysis from the Breathing Not Properly Multinational Study. Am J Kidney Dis 2003;41:571-9.

25. Takami Y, Horio T, Iwashima Y, Takiuchi S, Kamide K, Yoshihara F, et al. Diagnostic and prognostic value of plasma brain natriuretic peptide in non-dialysis-dependent CRF. Am J Kidney Dis 2004;44: 420-8.

26. Naganuma T, Sugimura K, Wada S, Yasumoto R, Sugimura T, Masuda C, et al. The prognostic role of brain natriuretic peptides in hemodialysis patients. Am J Nephrol 2002;22:437-44.

27. Sise ME, Baggett MV, Shepard JO, Stevens JS, Rhee EP. Case 17-2020: A 68-year-old man with covid-19 and acute kidney injury. $\mathrm{N}$ Engl J Med 2020;382:2147-56.

28. Chu KH, Tsang WK, Tang CS, Lam MF, Lai FM, To KF, et al. Acute renal impairment in coronavirus-associated severe acute respiratory syndrome. Kidney Int 2005;67:698-705.

29. Jung J, Park B, Hong S, Koh Y, Suh G, Jeon K, et al. Acute kidney injury in critically ill patients with pandemic influenza a pneumonia 2009 in Korea: A multicenter study. J Crit Care 2011;26:577-85.

30. Cheng Y, Luo R, Wang K, Zhang M, Wang Z, Dong L, et al. Kidney disease is associated with in-hospital death of patients with covid-19. Kidney Int 2020;97:829-38.

31. Diao B, Wang C, Wang R, Feng Z, Tan Y, Wang H, et al. Human kidney is a target for novel severe acute respiratory syndrome coronavirus 2 (SARS-CoV-2) infection. 2020 [Preprint].

32. Su H, Yang M, Wan C, Yi LX, Tang F, Zhu HY, et al. Renal histopathological analysis of 26 postmortem findings of patients with COVID-19 in China. Kidney Int 2020;98:219-27. 